\title{
Simulation and Fabrication of Integrated Polystyrene Microlens in Microfluidic System
}

\author{
Yiqiang Fan, Huawei Li, David Conchouso and Ian G. Foulds ${ }^{\mathrm{a}}$ \\ ${ }^{a}$ Electromechanical Microsystems \& Polymer Integration Research (EMPIRe) Group, Computer, \\ Electrical \& Mathematical Sciences \& Engineering Division (CEMSE), King Abdullah University of \\ Science and Technology (KAUST), Saudi Arabia; E-mail: yiqiang.fan@kaust.edu.sa
}

\begin{abstract}
This paper presents a simple and quick method to integrate microlens with the microfluidics systems. The polystyrene (PS) based microlens is fabricated with the free surface thermal compression molding methods, a thin PS sheet with the microlens is bonded to a PMMA substrate which contains the laser ablated microchannels. The convex profiler of the microlens will give a magnified images of the microchannels for easier observation. Optical simulation software is being used for the design and simulation of the microlens to have optimal optical performance with the desired focal length. A microfluidic system with the integrated PS microlens is also fabricated for demonstration.
\end{abstract}

Keywords: microlens, microfluidics, polystyrene

\section{INTRODUCTION}

In recent year, the interest of fabricate microlens keeps rising due to the wide applications in the display, optical processing, optical communication and data storage. Various fabrication methods of microlens has been developed[1-3] using photoresist reflow, laser ablation, injection molding and hot embossing. Most of these fabrication methods were complicated which require delicate devices and cleanroom facilities. In this paper, we proposed a simple free surface thermal compression molding method to fabricate the microlens and microlens array on polystyrene (PS) surface. Comparing to the previous high cost and complicated hot embossing methods such as silicon mold[4] or metal mold[5] stamping, in our fabrication process, we use the laser-patterned PMMA (polymethyl methacrylate) as the mold which was much more rapid and low cost.

Different materials such as polymers[6] and glasses[7] are being used for the fabrication of the microlens. Polymers are being widely used for the low cost and easy fabrication. Among these polymers, polystyrene is one of the most widely used materials in our daily life. Polystyrene has a high transmission of the visible light[8], which make PS very suitable for the lens material in optical devices. In our proposed fabrication method, we integrated the polystyrene microlens into the microfluidic system for point-of-care observation.

For the past two decades, there was a steady increase of research interest in microfluidic system for its potential application in chemical and biological field[9-11]. Polymers such as PDMS[12], PMMA[13] and PS[14] were widely used with different fabrication methods for microfluidic systems due to its low cost, transparency and bio-compatibility. The microfluidic technology had a bright prospect as it can perform an ordinary laboratory operations with a small volume of the reagents in a very short time. The volume of the reagents can be decreased from milliliters to microliters or even nanoliters while the reaction time can be reduced to several seconds or even less. In this study, we will integrate the PS microlens with the microfluidic system for the easy observation of the fluid flow or reaction in the microchannels. The fabrication method was easy accessible without delicate equipments which was suitable for most scientists and industrialists.

In this paper, polystyrene microlens were carefully designed and simulated, with the help of simulation result, we can optimize the design to make sure the lens has the minimum spherical aberration while maintain the distance from the object to the lens within the magnified range. PS microlens was fabricated using free-surface thermo-compression molding techniques with a laser cut PMMA mold which the details of the process will be explained in the followed section. 


\section{SIMULATION}

Design of the microlens was based on the simulation with the ZEMAX software. The microlens was fabricated using the free surface thermal compression method on a thin $(1.5 \mathrm{~mm})$ polystyrene sheet. In order to have the magnified virtual image for observation, the effective focal length for this single convex lens should be greater than $1.5 \mathrm{~mm}$. As shown in Figure 2, the variables are the radius of the lens and lens thickness. By controlling these two variables, we are trying to find an optimal design of the lens which has the focal length greater than $1.5 \mathrm{~mm}$ with relatively low aberration. The relations of the radius of the lens $(\mathrm{R})$, diameter of the lens $(\mathrm{D})$, thickness of the lens $(\mathrm{t})$, focal length $(f)$ and the reflective index (n) can be demonstrated using the following equations:

$$
R=\frac{D^{2}+4 t^{2}}{8 t}, \mathrm{f}=\frac{R}{n-1}
$$
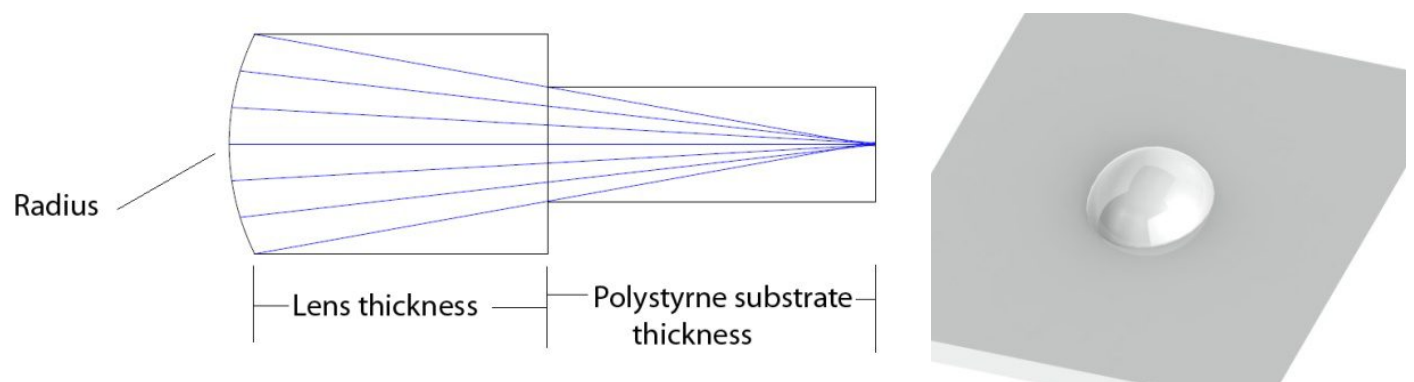

Figure 1. Schematic diagram of the lens design (plot of 2D and 3D).

The environment parameters setting before the simulation process were as following: The data of the refractive index was obtained from Kasarova's dispersion formula[15]. Three wavelengths $(0.486 \mu \mathrm{m}, 0.587 \mu \mathrm{m}, 0.656 \mu \mathrm{m})$ were selected from visible light range $(0.4-0.8 \mu \mathrm{m})$ to represent the visible light during the simulation process. Entrance pupil (aperture) was set at $1 \mathrm{~mm}$. Thickness of the polystyrene substrate was $1.5 \mathrm{~mm}$.

Effective focal length calculation result was shown in Figure 2a. We can see from the graph that the Effective focal length has an ideally positive linear relationship with the lens radius. The graph also illustrated the calculation result of effective focal length (EFFL) at three different lens thickness, the effective focal length will decrease as we increase the lens thickness. We can conclude from Figure 2a that in order to have a effective focal length longer than $1.5 \mathrm{~mm}$ we need to have a large lens radius with relatively small lens thickness. The relation of the back focal length and lens radius was shown in Figure $2 b$ which indicate the back focal length have a positive linear relationship with the change of lens radius. In our optimization process, we choose the effective focal length as the domain variable.

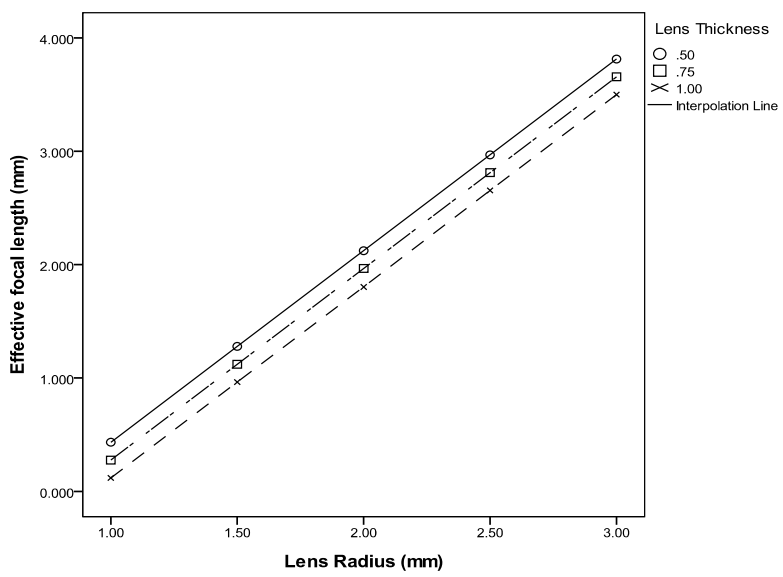

(a)

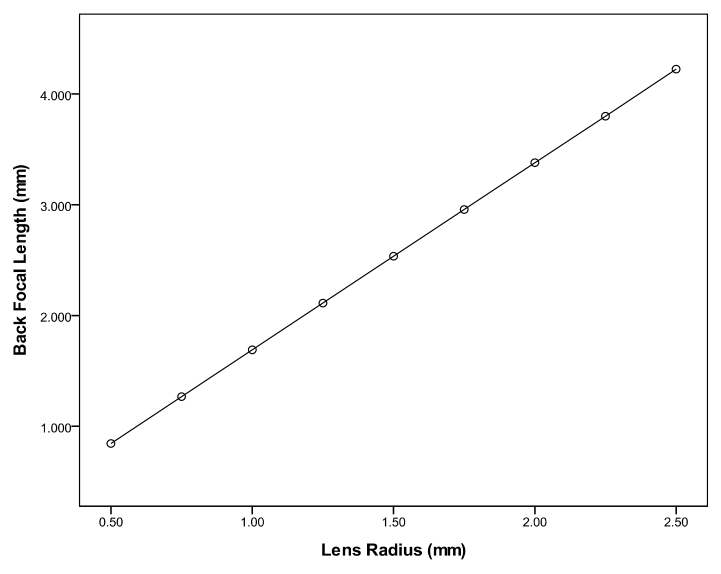

(b)

Figure 2. (a) The calculation result of the effective focal length at different lens radius with three different lens thickness. (b) The back focal length with different lens radius. 
Ray fan plots represent the transverse aberration as a function of the ray height in the pupil. Figure 3 showed the ray fan plot for transverse primary spherical aberrations of three rays. The rays were passing though the PS microlens with the wavelengths at $0.468 \mu \mathrm{m}, 0.568 \mu \mathrm{m}$ and $0.656 \mu \mathrm{m}$ respectively. Figure $3 \mathrm{a}$ and $3 \mathrm{~b}$ were the ray fan plot before and after the optimization of the microlens design. As indicated from the graph, the maximum aberration was decreased from \pm 50 $\mu \mathrm{m}$ to $\pm 20 \mu \mathrm{m}$. The initial setting of the radius and thickness of the lens were $1 \mathrm{~mm}$ and $1 \mathrm{~mm}$, after the optimization process, the radius was modified to $1.127 \mathrm{~mm}$ with a modified thickness of $1.456 \mathrm{~mm}$. The effective focus length after the optimization process was $1.904 \mathrm{~mm}$ which was ideally met our requirement for the focal length longer than $1.5 \mathrm{~mm}$.

Ray spot plot present ray distribution in the image plane. Figure 4 shown the ray spot plots of three rays with different wavelengths passing though the PS microlens before and after optimization process. As illustrated in the Figure 4a and $4 \mathrm{~b}$, the spherical aberration is significantly decreased after the optimization.

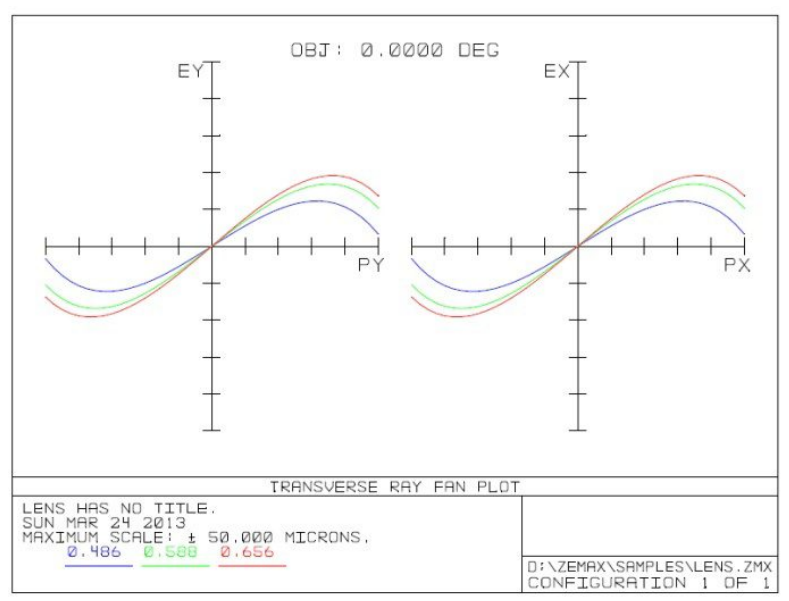

(a)

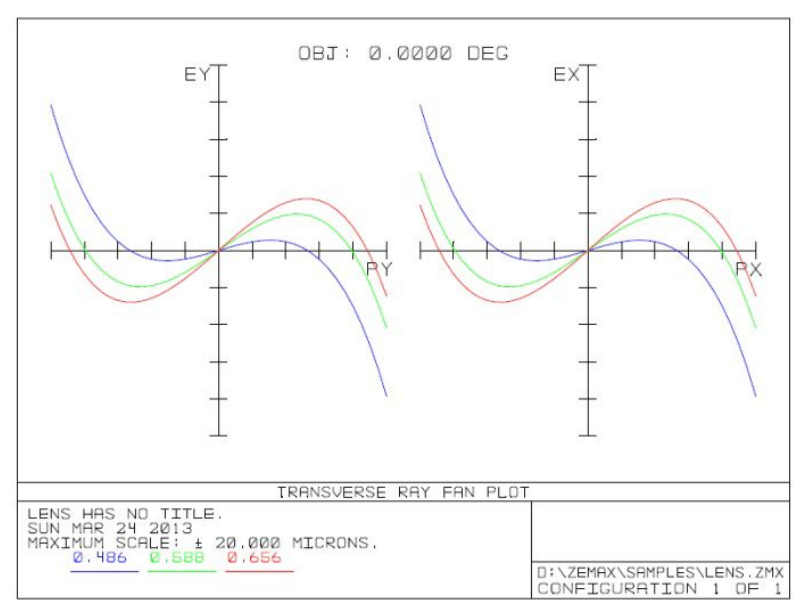

(b)

Figure 3. Ray fan plots for spherical aberration of three rays at different wavelengths in the visible range of light, before (a) and after (b) optimization process.

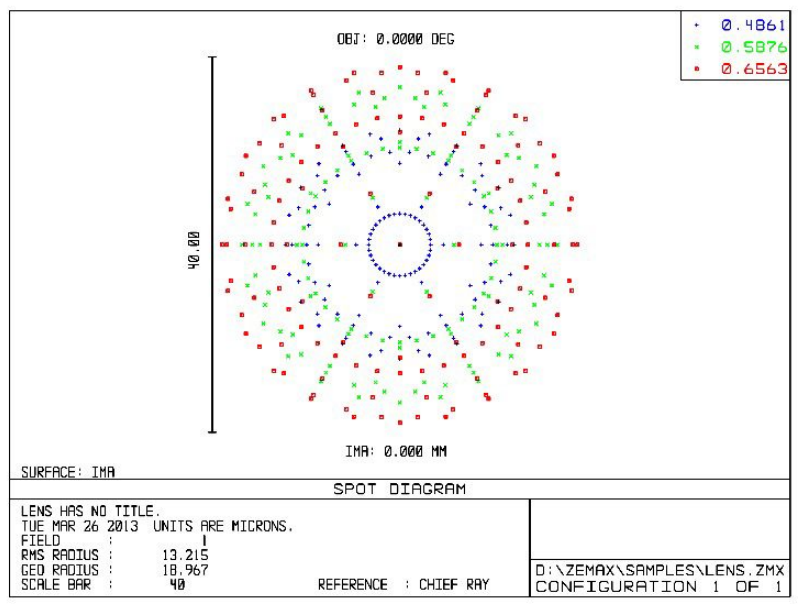

(a)

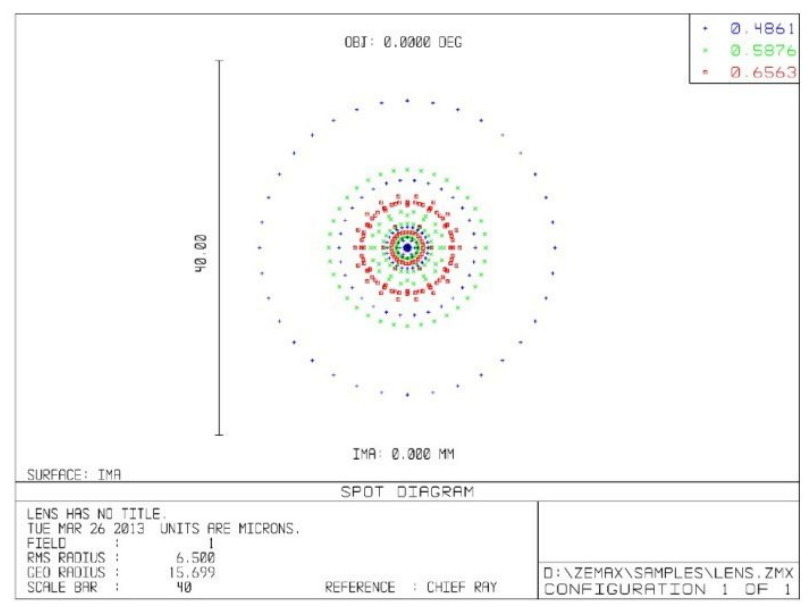

(b)

Figure 4. Ray spot plots for spherical aberration of three rays at different wavelengths in the visible range of light, before (a) and after (b) optimization process. 


\section{FABRICATION PROCESS}

A microfluidic device was fabricated for demonstration. This microfluidic device was a T-shape droplet generator with the integrated polystyrene microlens for easier observation.

The first step was using a commercial laser system (Universal PLS6.75) to fabricate the microchannels on a $2 \mathrm{~mm}$ thick PMMA (YNG GUANG Corp) sheet (shown in Figure 5a). The laser power was $37.5 \mathrm{~W}$ with the scanning speed of 150 $\mathrm{mm} / \mathrm{s}$. Another $2 \mathrm{~mm}$ thick PMMA sheet was also laser ablated for though holes which will be used as the mold for the following free surface thermal compression molding process (Figure 5b). The dimension of the though holes were based on the previous mentioned simulation process which will decide the dimension of the microlens after the molding process. PMMA has a higher glass transition temperature $\left(\sim 110^{\circ} \mathrm{C}\right)$ than polystyrene $\left(\sim 90^{\circ} \mathrm{C}\right)$, which enable the PMMA to be used as the mold for the thermal compression molding process of the PS microlens.

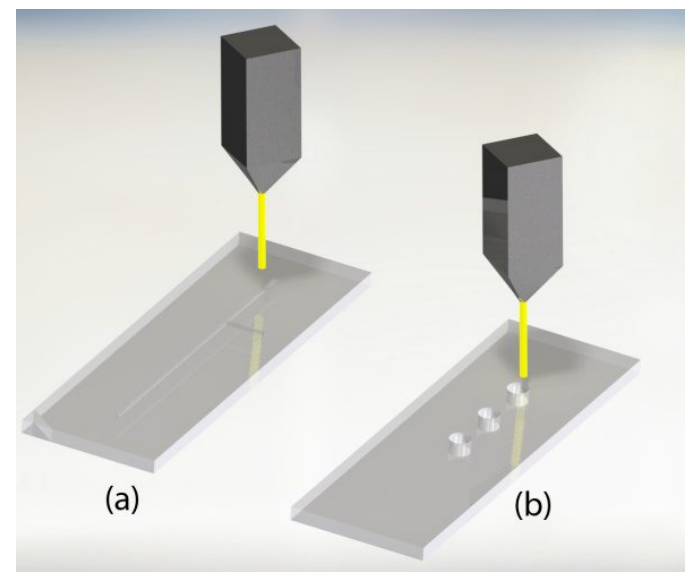

Figure 5. Schematic diagram for the laser fabrication on the $2 \mathrm{~mm}$ thick PMMA sheet. (a): laser ablation for microchannels. (b): Laser ablated PMMA mold for the following compression molding process.

The free surface thermal compression bonding process was performed using the INSTRON dual column testing system. Figure 6 shows the system setup, the system had a environmental chamber which can control the environment temperature. The lower and upper arm were controlled by the software to achieve the desired pressure. The prefabricated PMMA mold (Figure 7a) was then attached to a $1.2 \mathrm{~mm}$ thick PS sheet (molecular weight of $267.8 \mathrm{kDa}$ from Goodfellow Cambridge Limited, England). The PMMA and PS sheets were then sandwiched between two arms inside the environmental chamber and then start the bonding process. With the bonding force setting at $800 \mathrm{~N}$ for 60 minutes at $130^{\circ} \mathrm{C}$. The microlens will formed on the surface of polystyrene (Figure $7 \mathrm{~b}$ ).

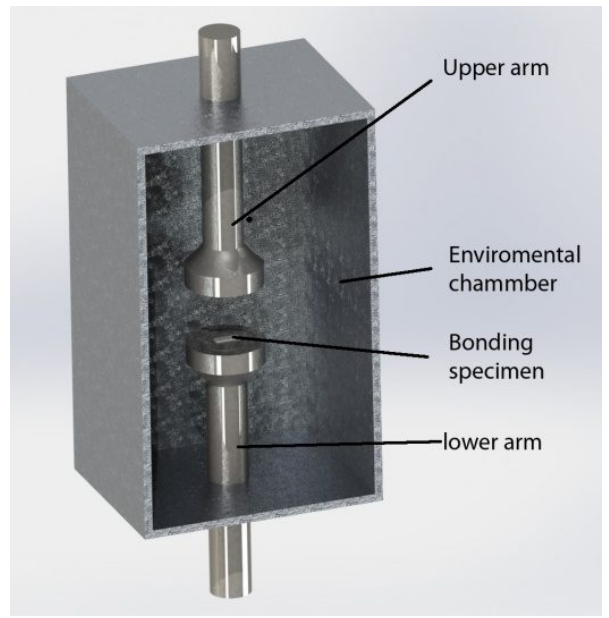

Figure 6. Schematic for the bonding system setup. The system consist of a environmental chamber and two arms for compression. 
The final step was bonding the $1.2 \mathrm{~mm}$ thick PS sheet with microlens to the $2 \mathrm{~mm}$ thick PMMA sheet which contained microchannels (shown in Figure 7d). The same system as shown in Figure 6 was used for the final bonding process. With the bonding temperature at $120^{\circ} \mathrm{C}$ and bonding force of $400 \mathrm{~N}$ for an hour, the PS and PMMA sheet were bonded. After connecting the inlet and outlet ports with pipes (TYGON Tubing), this T-shaped droplet generator was ready for testing.

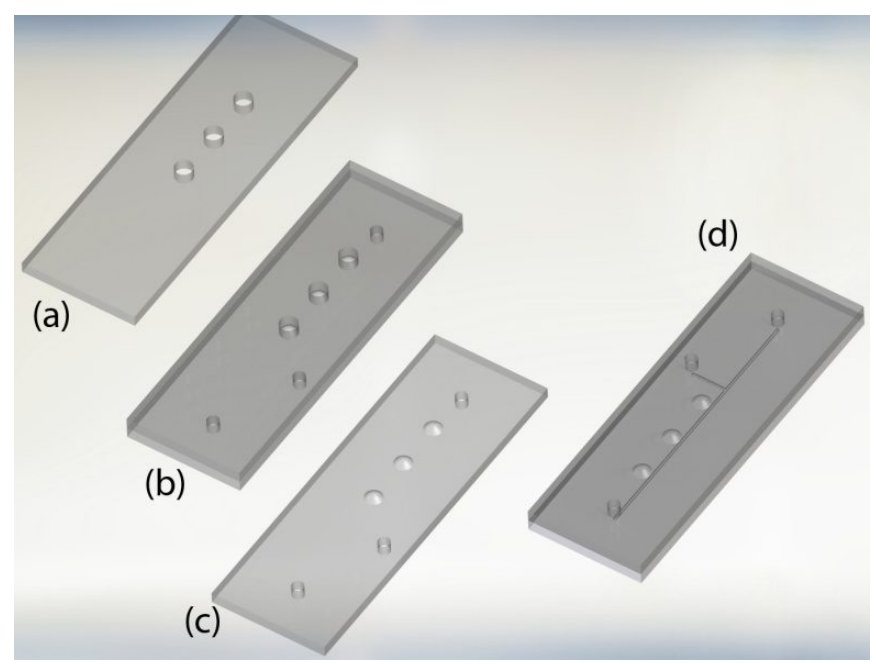

Figure 7. Fabrication process for the microfluidic device with integrated microlens.

\section{RESULT AND DISCUSSION}

For demonstration, a T-shape droplet generator was design and fabricated. The system setup for the chip testing was shown in Figure 8, the two inlet flow (oil and water) were controlled by two syringe pumps (Chemyx Fusion200) respectively. The liquid flow inside the microchannels was observed by the microscope (ZEISS Examiner D1). In this Tshaped droplet generator, force created by the cross-flowing continuous phase (mineral oil) periodically break off droplets as the dispersed phase (water dye in red) was injected into the main channel. The enlarged image in Figure 8 shows the droplet generator with the integrated PS microlens.

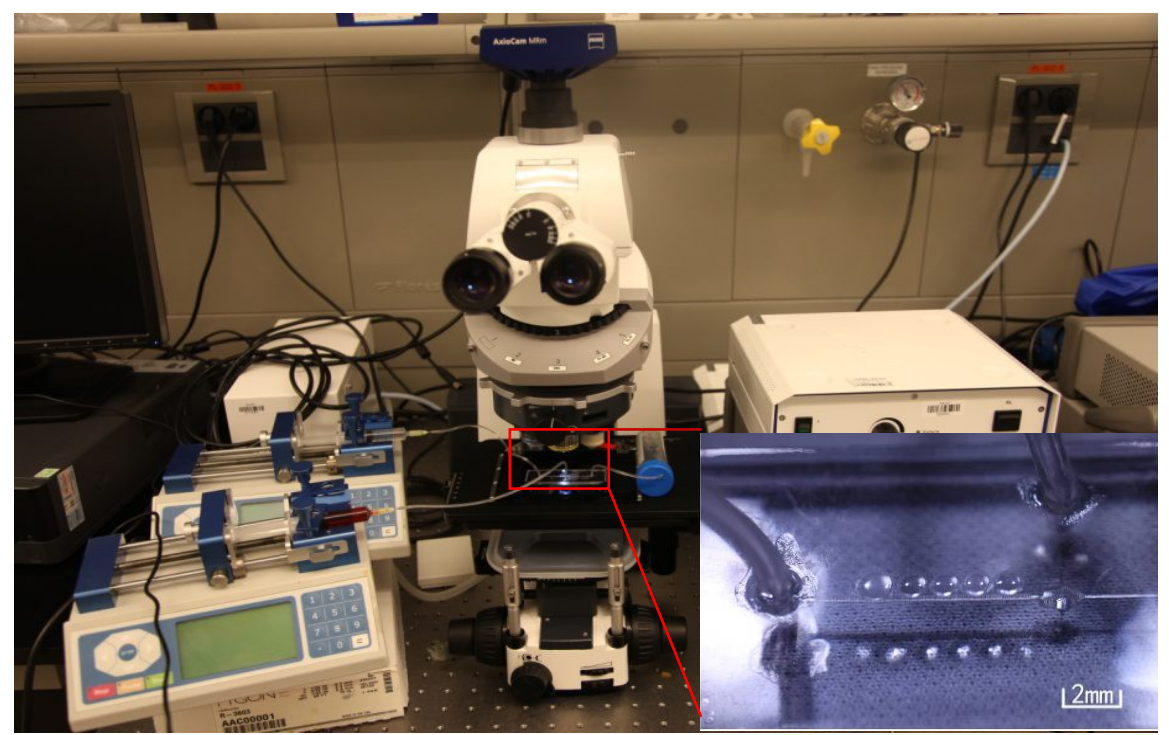

Figure 8. System setup for the testing of T-shaped droplet generator. 
Images taken using the microscope showed the channel under the polystyrene microlens was obviously magnified. The two images in Figure 9 were captured with the same microscope magnification (10x) where Figure 9a was taken through a polystyrene microlens, showing the image of the microchannel was enlarged by $\sim 2 \mathrm{x}$ when compared to Figure $9 \mathrm{~b}$ taken of a normal channel without the microlens. The profiler of the PS microlens was obtained using the profilometer (Ambios XP-200). Figure 10 shows the result of the lens profile measurement. The diameter of the lens was about 1.2 $\mathrm{mm}$ with a height of $1.6 \mathrm{~mm}$, which was highly matched to the optimized simulation result of $1.127 \mathrm{~mm}$ in diameter and $1.456 \mathrm{~mm}$ in height. SEM images (Quanta 600) of the PMMA mold and PS microlens were shown in Figure 11.
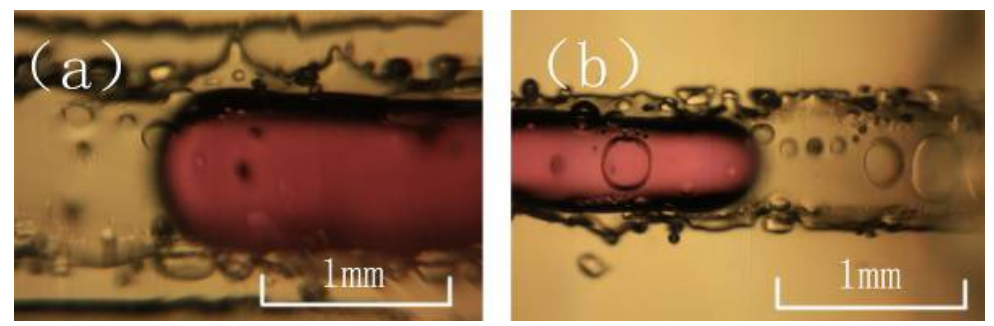

Figure 9. Fabrication process for the microfluidic device with integrated microlens.

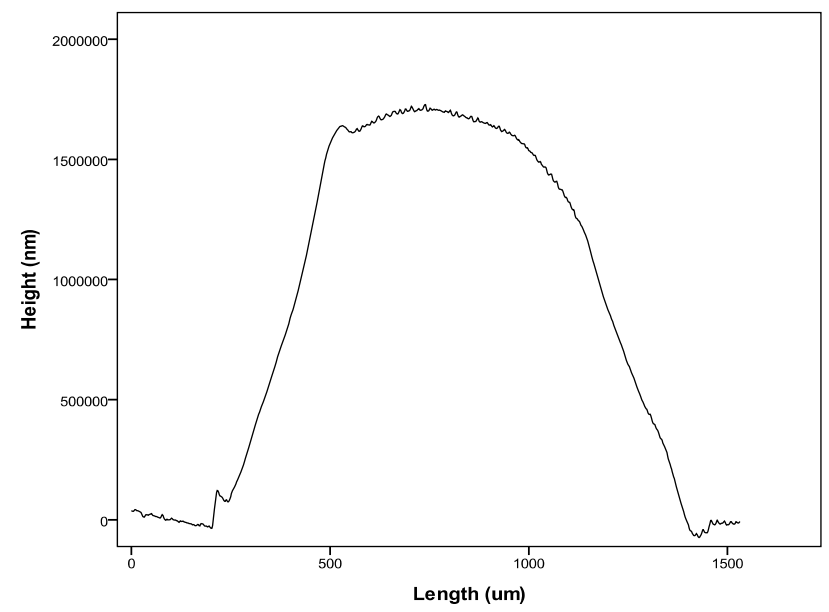

Figure 10. Profile measurement of the polystyrene microlens. The lens diameter was $1.2 \mathrm{~mm}$ with a height of $1.6 \mathrm{~mm}$.

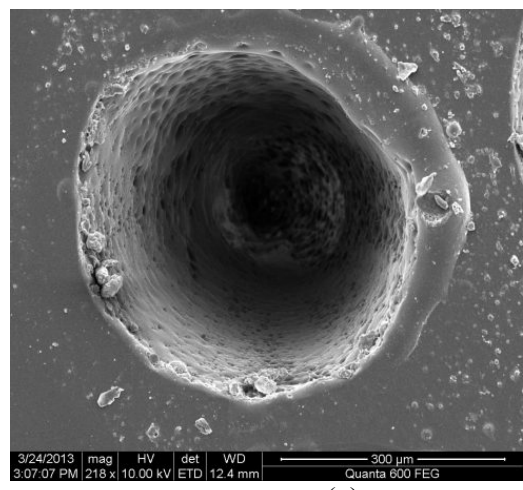

(a)

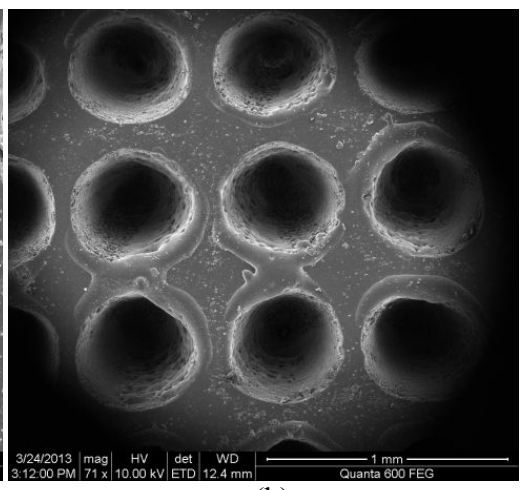

(b)

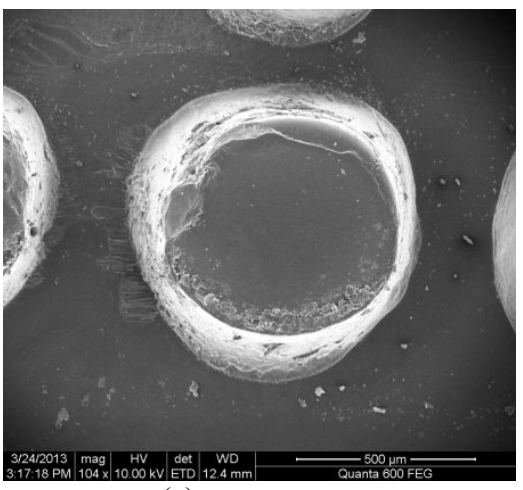

(c)

Figure 11. SEM images.(a)(b): Laser fabricated PMMA mold. (c): Polystyrene microlens fabricated after the molding. 


\section{CONCLUSION}

In this study, we present a novel method for the integration of microlens and microfluidic devices with the optimized lens design. The software was used for the simulation and optimization of the microlens design. This study was also present a simple and rapid free surface thermo compression molding process for the formation of polystyrene microlenses. The experimental result shows the microlens have the function of enlarging the images for easy observation of the liquid flow inside the microchannels. This simple and rapid method of fabricating microfluidic devices with integrated microlens has a widely applications on point-of-care testing in the biology and chemical field.

\section{REFERENCES}

[1] J. Chen, W. Wang, J. Fang et al., "Variable-focusing microlens with microfluidic chip," Journal of Micromechanics and Microengineering, 14(5), 675-680 (2004).

[2] T. Krupenkin, S. Yang, and P. Mach, “Tunable liquid microlens," Applied Physics Letters, 82(3), 316 (2003).

[3] S. Park, Y. Jeong, J. Kim et al., "Fabricaton of Poly(dimethylsiloxane) Microlens for Laser-Induced Fluorescence Detection," Japanese Journal of Applied Physics, 45(6B), 5614-5617 (2006).

[4] C. Y. Chang, S. Y. Yang, L. S. Huang et al., "Fabrication of plastic microlens array using gas-assisted micro-hotembossing with a silicon mold," Infrared Physics \& Technology, 48(2), 163-173 (2006).

[5] C.-Y. Chang, S.-Y. Yang, and M.-H. Chu, "Rapid fabrication of ultraviolet-cured polymer microlens arrays by soft roller stamping process," Microelectronic Engineering, 84(2), 355-361 (2007).

[6] T.-K. Shih, C.-F. Chen, J.-R. Ho et al., "Fabrication of PDMS (polydimethylsiloxane) microlens and diffuser using replica molding," Microelectronic Engineering, 83(11-12), 2499-2503 (2006).

[7] C. Y. Chang, S. Y. Yang, and J. L. Sheh, "A roller embossing process for rapid fabrication of microlens arrays on glass substrates," Microsystem Technologies, 12(8), 754-759 (2006).

[8] Y. Q. Fan, H. W. Li, and I. G. Foulds, "Fabrication of Microlens and Microlens Array on Polystyrene Using $\mathrm{CO}<$ sub $>2</$ sub $>$ Laser," Advanced Materials Research, 403-408, 3350-3353 (2011).

[9] S. Haeberle, and R. Zengerle, "Microfluidic platforms for lab-on-a-chip applications," Lab on a Chip, 7(9), 1094 (2007).

[10] S.-Y. Teh, R. Lin, L.-H. Hung et al., "Droplet microfluidics,” Lab on a Chip, 8(2), 198 (2008).

[11] G. M. Whitesides, "The origins and the future of microfluidics," Nature, 442(7101), 368-373 (2006).

[12] S. Seiffert, J. Dubbert, W. Richtering et al., "Reduced UV light scattering in PDMS microfluidic devices," Lab on a Chip, 11(5), 966 (2011).

[13] Y. Fan, Y. Liu, H. Li et al., "Printed wax masks for 254 nm deep-UV pattering of PMMA-based microfluidics," Journal of Micromechanics and Microengineering, 22(2), 027001 (2012).

[14] A. S. Johnson, K. B. Anderson, S. T. Halpin et al., "Integration of multiple components in polystyrene-based microfluidic devices part I: fabrication and characterization," The Analyst, 138(1), 129 (2013).

[15] S. N. Kasarova, N. G. Sultanova, C. D. Ivanov et al., "Analysis of the dispersion of optical plastic materials," Optical Materials, 29(11), 1481-1490 (2007). 